John Andraos*

\title{
Aiming for a standardized protocol for preparing a process green synthesis report and for ranking multiple synthesis plans to a common target product
}

https://doi.org/10.1515/gps-2019-0048

Received April 01, 2019; accepted May 29, 2019.

Abstract: This paper proposes a standardized format for the preparation of process green synthesis reports that can be applied to chemical syntheses of active pharmaceutical ingredients (APIs) of importance to the pharmaceutical industry. Such a report is comprised of the following eight sections: a synthesis scheme, a synthesis tree, radial pentagons and step E-factor breakdowns for each reaction step, a tabular summary of key material efficiency step and overall metrics for a synthesis plan, a mass process block diagram, an energy consumption audit based on heating and cooling reaction and auxiliary solvents, a summary of environmental and safety-hazard impacts based on organic solvent consumption using the Rowan solvent greenness index, and a cycle time process schedule. Illustrative examples of process green synthesis reports are given for the following pharmaceuticals: 5-HT2B and 5-HT7 receptors antagonist (Astellas Pharma), brivanib (Bristol-Myers Squibb), and orexin receptor agonist (Merck). Methods of ranking synthesis plans to a common target product are also discussed using 6 industrial synthesis plans of apixaban (Bristol-Myers Squibb) as a working example. The Borda count method is suggested as a facile and reliable computational method for ranking multiple synthesis plans to a common target product using the following 4 attributes obtained from a process green synthesis report: process mass intensity, mass of sacrificial reagents used per kg of product, input enthalpic energy for solvents, and Rowan solvent greenness index for organic solvents.

Keywords: green chemistry education; green engineering education; process chemistry; process mass intensity; sustainability metrics

* Corresponding authors: John Andraos, CareerChem, Toronto, ON M3B 2W4 Canada, e-mail: c1000@careerchem.com

\author{
Abbreviations: \\ 5-HT2B = 5-hydroxytryptamine-2B receptor \\ 5-HT7 = 5-hydroxytryptamine-7 receptor \\ $\mathrm{ABP}=$ acidification-basification potential \\ $\mathrm{AE}=$ atom economy \\ API $=$ active pharmaceutical ingredient \\ aqua $=$ aquatic toxicity, $\mathrm{mg} / \mathrm{L}$ for $96 \mathrm{~h}$ \\ $\mathrm{BCP}=$ bioconcentration potential, unitless \\ cPMI = cumulative process mass intensity \\ $E=$ E-factor
}

EATOS $=$ Environmental Assessment Tool for Organic Synthesis

EDAC = 3-ethyl-1-(3-dimethylaminopropyl) carbodiimide

FLASC $=$ Fast Life Cycle Assessment of Synthetic Chemistry

$\mathrm{FP}=$ flash point, degrees $\mathrm{K}$

$\mathrm{GWP}=$ global warming potential, unitless

$\mathrm{I}=$ intermediate

IEE = input enthalpic energy, $\mathrm{kJ}$ per $\mathrm{kg}$ product

$j=$ refers to $j^{\text {th }}$ step in a synthesis plan

$K$ = number of synthesis plans to a common target product

$\mathrm{K}_{\mathrm{oc}}=$ soil adsorption coefficient, unitless

$\mathrm{K}_{\mathrm{ow}}=$ octanol-water partition coefficient, unitless

LC50 = lethal concentration required to kill $50 \%$ of population, $\mathrm{g} / \mathrm{m}^{3}$ for $4 \mathrm{~h}$

LD50 = lethal dose required to kill $50 \%$ of population, $\mathrm{mg} /$

$\mathrm{kg}$ body weight

$m$ = number of attributes used in a ranking algorithm

$M=$ metric

$\mathrm{MRP}=$ material recovery parameter

$N=$ number of reaction steps

ODP = ozone depletion potential, unitless

$\mathrm{OEL}=$ occupational exposure limit, $\mathrm{ppm}$

$O S I=$ overall solvent index

OPSI = overall pharmaceutical solvent index

$\mathrm{P}=$ target product in synthesis plan

$\mathrm{PER}=$ persistence potential, unitless

$\mathrm{PMI}=$ process mass intensity

$\mathrm{Q}=$ quotient referring to risk phrases, unitless

$r=$ rank number for a plan

$\mathrm{R}=$ reagents 
$\mathrm{RME}=$ reaction mass efficiency

RSGI = Rowan solvent greenness index, $\mathrm{kg}$

$\mathrm{SD}=$ skin dose, $\mathrm{mg}$

$\mathrm{SF}=$ stoichiometric factor

$\mathrm{SFP}=$ smog forming potential, unitless

$\mathrm{SR}=$ sacrificial reagents

$\mathrm{STY}=$ space-time-yield, $\mathrm{kg} / \mathrm{m}^{3} / \mathrm{h}$ or $\mathrm{kg} / \mathrm{L} / \mathrm{h}$

VTO = volume time output, $\mathrm{h} \mathrm{m}^{3} / \mathrm{kg}$

$\mathrm{W}=$ waste

$\mathrm{Y}=$ yield

\section{Introduction}

The pharmaceutical industry has been at the forefront of implementing green chemistry principles in the manufacture of active pharmaceutical ingredients over the last two decades [1-11]. Along with this development there has also been parallel intensive research into parameterizing measures that can gauge the progress of waste reduction, energy consumption, and reduced environmental and safety-hazard impacts when carrying out reaction and synthesis plan optimization. Very recently a detailed glossary of so-called green metrics vocabulary has been compiled covering 25 years of literature on this subject [12]. At this mature stage of research in the field, what is needed to implement green chemistry practice as a matter of routine in process chemistry optimization development is a standardized protocol to describe the "green" attributes of various shades for a given synthesis plan in an easy-to-understand and visually appealing manner that gets across the main highlights quickly to members of a team of professionals having various disciplinary backgrounds but who are collectively engaged in the process chemistry enterprise. Essentially, what is needed is a report that contains and communicates the vital information that chemists and engineers need to know in order to implement green chemistry principles in their work. Traditionally, declarations of "efficient syntheses" in the literature have mainly gravitated to merits with respect to some measure of material efficiency such as fewer reaction steps, increased overall yield, increased Green Aspiration Level [13], or decreased E-factors [14] and process mass intensities [15]. These measures of synthesis optimization are all obvious, well understood, and well implemented in the literature. However, they completely neglect other factors that are equally important in tracking and ultimately ranking continued improvements in process synthesis design such as environmental and occupational impact, and energy consumption. Limitations of massmetrics-only analysis in decision-making and ranking have been discussed previously [16]. Over the years more holistic analyses of synthesis plans to pharmaceutical targets have addressed these deficits by including environmental and safety-hazard assessments such as Edwards-Lawrence algorithm [17], EATOS [18-20], EcoScale [21], Green Star [22], and FLASC [23]. Energy metrics are by far the least implemented and discussed in the context of process chemistry optimization in the pharmaceutical industry as evidenced by the massmetric-only PMI calculators available on the ACS Green Chemistry Institute Pharmaceutical Roundtable website [24]. Furthermore, a number of methods appearing in the literature, such as FLASC, are proprietary in-house algorithms specific to the needs of the pharmaceutical company advancing them and are not readily accessible to the wider chemistry community to use freely. In this work we present a simplified yet comprehensive "process green synthesis report" protocol that aims to achieve the following goals: (a) present a succinct summary analysis of a given process synthesis according to mass, impact, and energy metrics that are deemed to be essential in assessments of efficiency so that holistic decision-making can be accomplished; (b) present accompanying simple visual aids to each of these metrics analyses to quicken the identification of metric pros and cons for a given process synthesis; and (c) present all of this information in a manner that can be understood by both chemists and chemical engineers so that both groups of scientists who have different training and technical vocabulary backgrounds can come together and communicate in order to solve a given optimization problem while implementing green chemistry principles. Therefore, with this tool in hand, any chemist or chemical engineer can quickly glean the strengths and weaknesses of a given synthesis plan and then act on those results to initiate further improvements that address any shortcomings and "hot spots" with respect to material and energy consumption, and environmental and safety-hazard issues that have been identified.

We first present and describe the various sections of such a report and then illustrate its use by showing example reports for the industrial manufacture of three pharmaceuticals shown in Figure 1: 5-HT2B and 5-HT7 antagonist [25], brivanib [26-29], and orexin receptor agonist [30]. The pros and cons of each synthesis are discussed with respect to the three categories of green metrics mentioned above. These three selected pharmaceuticals are representative of the kinds of syntheses that are usually employed in scaled-up drug manufacture. Important common characteristics of such plans are isolation of solid derivatives that can be purified 


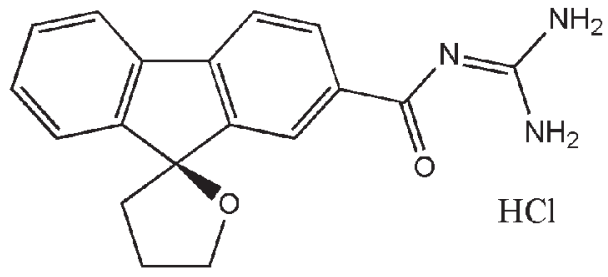

5-HT2B and 5-HT7 antagonist

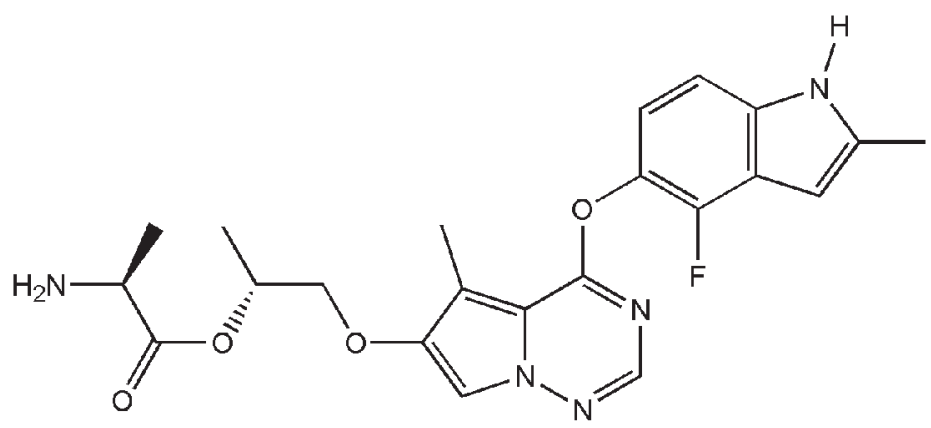

brivanib<smiles>Cc1ccc(-c2ncccn2)c(C(=O)N2C[C@H](COc3ccc(F)cn3)CC[C@H]2C)c1</smiles>

orexin receptor agonist

Figure 1: Chemical structures of the three pharmaceuticals examined in this work.

by crystallization methods and telescoped reaction steps to reduce solvent demand in workup procedures. The synthesis of the 5-hydroxytryptamine antagonist is a linear plan involving a classical resolution step via a diastereomeric salt intermediate in order to obtain the desired enantiomeric product. The ring construction mapping of the spiro ether in the final product structure is [3+2] and the ring construction strategy to construct this 5 -membered ring is [5+0]. The synthesis of brivanib is convergent and involves constructing the indole and pyrrolo[2,1-f][1,2,4]triazine ring moieties in separate branches and then coupling these rings via an ether linkage. The ring construction mappings in the final product structure for these rings are $[3+2]$ and $[(3+2)+$ $(2+1+2+1)]$, respectively. The indole ring is made via a [5+0] cyclization involving a tandem nitro to amino group reduction and subsequent intramolecular cyclization of the amino group onto a ketone moiety with concomitant elimination of water. The pyrrolotriazine fused bicyclic ring system is made via an initial [3+2] cyclization forming the pyrrole ring moiety by coupling ethyl crotonate and tosylmethyl isocyanide, and then the triazine ring moiety is built sequentially via a [4+2] cycloaddition involving formamide and a 1-amino-2-carboxyethylpyrrole intermediate. The plan involves well-known reactions and the chiral alkoxy moiety is introduced via tandem ester deprotection affording a free alkoxide which subsequently ring opens (R)-propylene oxide from the less hindered side. The second chiral moiety is introduced via esterification of an N-benzyloxycarbonyl protected D-alanine reagent. The orexin receptor synthesis is also convergent and involves a biotransformation involving a transamination reaction between $\mathrm{D}$-alanine and a ketone intermediate mediated by D-glucose. This synthesis also involves a classical resolution step using (+)-10-camphorylsulfonic acid as resolving agent. The central piperidine ring is constructed via an intramolecular [6+0] cyclization forming a lactam intermediate. The ring construction mapping in the final product structure for this ring is $[3+2+1]$.

When there are multiple synthesis plans to a common target pharmaceutical, the question of appropriately ranking them according to the same mass, impact, and energy metrics described in their respective process green synthesis reports needs to be addressed. This second important problem has been tackled before using vector magnitude ratio determination (VMR) [12] and order theory via partially ordered set (poset) analysis applied to reaction and synthesis plan comparisons 


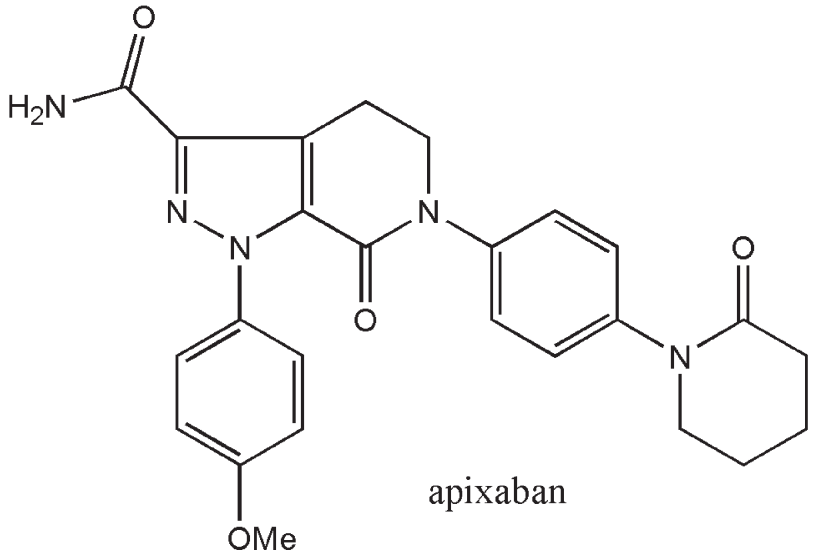

Figure 2: Chemical structure of apixaban.

[31]. In the last section of this work we advance a much simpler computational method based on the Borda count positional voting procedure [32,33]. We illustrate the results of all three ranking methods on 6 industrial plans to apixaban [34-39] shown in Figure 2 and discuss the merits and limitations of each method. Specifically, we show that the Borda count method yields the same ranking results as the poset method using simpler and faster computation, but are different from the results of the inferior VMR method.

\section{Process green synthesis report}

In this section we itemize and briefly describe the different parts of the process green synthesis report.

\subsection{Part 1 - Synthesis scheme}

A synthesis scheme is the primary piece of information that can be written down for how a particular target molecule can be put together. Traditionally, it is depicted as a sequence of intermediate structures interspersed with reaction arrows having only reagents and other reaction conditions depicted above those arrows. In the present formulation satisfying the needs of tracking what is going on over the course of a synthesis plan for the purposes of implementing green chemistry principles, the synthesis scheme consists of balanced chemical equations showing reaction by-products in each step below the reaction arrows and molecular weights of all intermediates and target product in addition to the traditional chemical structures of all isolated and telescoped intermediates. Particular attention is paid to drawing all structures of intermediates along the way in the same structural aspect to facilitate the tracking of which construction bonds are made over the course of the entire synthesis plan. Typically the way this is achieved is by deciding on a structural aspect for the final target product and then working backwards stepby-step toward the starting materials in the first step in such a way that the intervening intermediates are drawn in the same structural aspect so that the orientation of each fragment is preserved along the way. This technique in drawing structures greatly facilitates the visual reading of a scheme and allows for easy determination of ring construction strategies employed and ring mapping in the final product structure. Hence, for the chemical structure of the final target product, a target bond map is also given highlighting all synthesis connectivity bonds, particularly those involved in ring construction mappings. This latter piece of information is a new add-on that visually depicts synthesis strategy in two ways. Firstly, the target bond mapping allows for the tracing of the pieces of the starting materials that get incorporated into the product structure, which then in turn yields estimates of the atom and mass fractions of those input reagents. Secondly, the step-bystep tracing of target bonds throughout a synthesis plan from beginning to end allows for pinpointing when and how a given ring system was made along the way over the course of the plan. For example, if a 6-membered ring in the product structure is highlighted by two target bonds showing a [4+2] mapping, this could mean either that the 4-atom fragment is first linked to the 2-atom fragment in one step and then this is followed by a [6+0] cyclization in a later step, or that one step involves a simultaneous [4+2] cyclization of the two fragments. So, for the same [4+2] ring mapping we have two possible kinds of strategies that could be employed, a stepwise [6+0] cyclization or a direct [4+2] cyclization. The following general notation is used to describe a ring construction strategy for a target product having two rings $\mathrm{A}$ and $\mathrm{B}: \stackrel{[a+b]}{\longrightarrow} A \stackrel{[c+d]}{\longrightarrow} B_{k}$, where it is implied that an $[a+b]$ cyclization takes place in step $j$ to make ring $\mathrm{A}$, and $\mathrm{a}[c+d]$ cyclization takes place in step $k$ to make ring B. If the two rings comprise a fused bicyclic system, say [4.3.0], and are made simultaneously in a single step $i$, then the following notation is used to show how the rings A and B are made: $\stackrel{\left[(a+b)_{A}+(c+d)_{B}\right]}{\rightarrow}[4 \text {.3.0. }]_{i}$.

\subsection{Part 2 - Synthesis tree}

The depiction of synthesis plans as synthesis trees has been advanced previously [40]. It is a convenient diagram that compactly includes several pieces of key data about a synthesis plan. The following information can be readily gleaned from a synthesis tree: number of reaction steps, 
number of reagents used, stoichiometric coefficients of all reagents, molecular weights of intermediates and final target product, molecular weights of reagents, number of branches, step reaction yields, and number of convergent steps.

\subsection{Part 3-Radial pentagons and step E-factor breakdown}

Radial pentagons were advanced as an easy visual aid to gauge the material performance of an individual chemical reaction [41] once the roles and masses of all input materials are identified. The following five variables are depicted in radial pentagons: atom economy $(\mathrm{AE})$, reaction yield, inverse of stoichiometric factor (SF) indicating excess reagent consumption, material recovery parameter (MRP) indicating auxiliary material consumption, and reaction mass efficiency (RME). The RME is the numerical product of the four stated variables. Radial pentagons that have radial lengths close to 1 (towards the perimeter) are deemed green and those that have radial lengths close to 0 (towards the centre) are deemed non-green. The waste output production is depicted as a step E-factor pie chart showing percent E-factor contributions arising from E-kernel (waste by-products), E-excess (excess reagent consumption), E-rxn solvent (reaction solvent consumption), E-cat (catalyst consumption), E-workup (workup material consumption), and E-purif (purification material consumption). Each of these E-factors is found by dividing the mass of the contributing material by the mass of the final target product for a given reaction.

\subsection{Part 4 - Step and overall material metrics summary}

A table is presented that summarizes the following reaction step parameters: \% atom economy, \% reaction yield, E-kernel, E-excess, E-cat, E-workup, E-purif, E-overall, and PMI. These parameters are determined using the REACTION Excel spreadsheet $[42,43]$ described previously. In addition, the corresponding material efficiency metrics for the overall synthesis performance are given at the bottom of the above table. These overall parameters are determined using the SYNTHESIS Excel spreadsheet [42,43]. Cumulative PMI parameters are determined using the cumulative PMI calculator spreadsheet [44]. There is always consistent agreement between the overall PMI obtained from the SYNTHESIS spreadsheet and the cumulative PMI calculator. All material efficiency metrics calculated in this section are determined for a $1 \mathrm{~kg}$ basis mass of target product. Below this summary table positive and negative attributes are summarized identifying key steps that are best performers and worst performers, respectively according to each of the metrics shown in the summary table. For easy visual inspection, these are colour-coded as green and red.

A second table is given itemizing a list of scaled masses of reagents used to manufacture a $1 \mathrm{~kg}$ basis mass of target product including identification of masses of reagents corresponding to sacrificial reagents. Sacrificial reagents are those that do not contribute any atoms to the final product structure over the course of the synthesis plan and arise mainly in protection-deprotection steps and oxidation correction steps. Once the sacrificial reagents are identified, the mass fraction of sacrificial reagents is calculated with reference to the total mass of reagents employed in the synthesis as given in the synthesis tree diagram in Part 3. Such a fraction can also be correlated to the target bond mapping diagram given in the synthesis scheme shown in Part 1. Generally, materially efficient syntheses, in addition to having high overall yields and employing high atom economy transformations, also have low values for the mass fraction of sacrificial reagents. The last column in this table itemizes the reaction classification type for each reaction step in the synthesis plan.

\subsection{Part 5 - Mass process block diagram}

This kind of diagram is of high interest to chemical engineers who wish to track the input material throughput of all ingredients required and all waste output materials produced over the course of executing a synthesis plan. The purpose and merits of block diagrams and process flow sheets to depict industrial processes are nicely described by Jiménez-González and Constable [11]. However, traditional block diagrams usually show the sequence of unit operations but do not always show mass amounts of materials used and certainly do not show any green metrics. In this presentation these shortcomings are addressed. Therefore, the mass amounts shown in this kind of diagram advanced in this work correspond to the synthesis of a basis mass of $1 \mathrm{~kg}$ of target product consistent with the summary table of material efficiency metrics discussed in Part 4. Figure 3 shows template diagrams that pertain to single reaction steps in a mass process block diagram for a linear synthesis plan. Convergent reactions are highlighted in blue colour for easy identification in complex synthesis plans. Reagents are designated as $R$, intermediates are designated as 


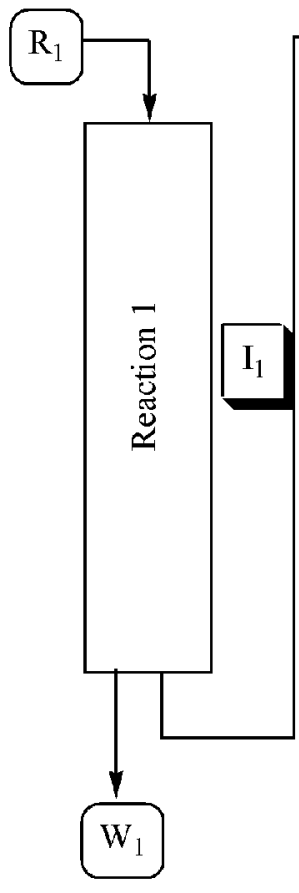

A

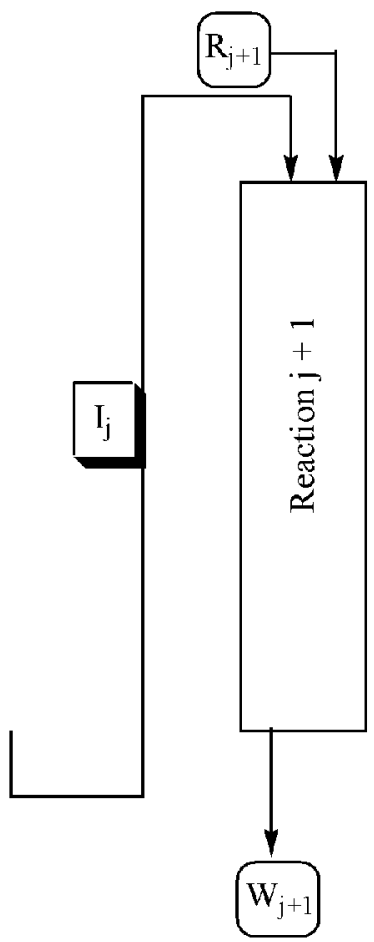

B

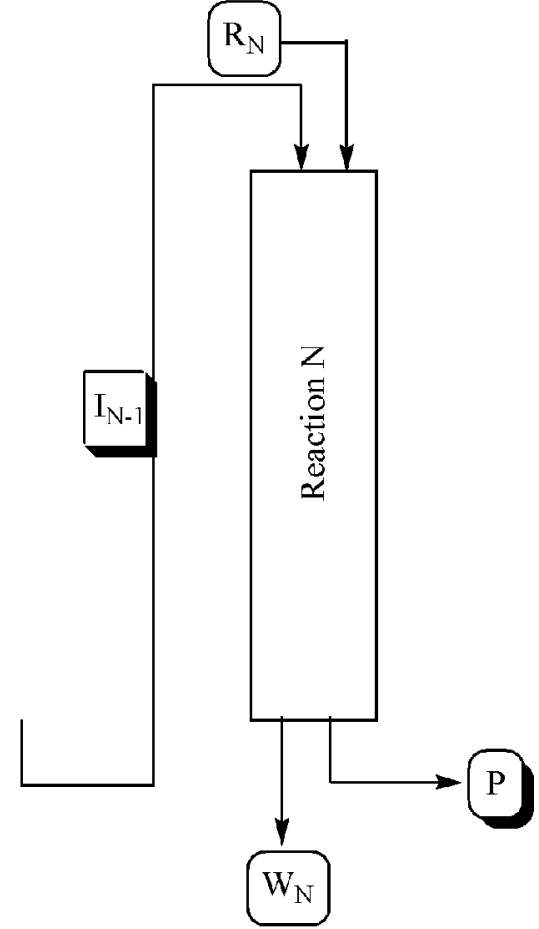

C

Figure 3: Template used to depict single chemical reactions in the construction of a mass process block diagram: (A) first reaction step; (B) $(j+1)^{\text {th }}$ reaction step; and (C) last reaction step in an $N$-step linear synthesis plan.

I, and wastes are designated as W. The block diagram itemizes masses of all reagents used, masses of isolated intermediates along the way, masses of waste by-products, total mass of waste from organic solvents, total mass of waste from aqueous solvents, and total mass of waste from solid inorganics (salts, catalysts, drying agents, filtering agents, chromatographic agents). At the end of the mass process block diagram there is also an embedded summary table of step and overall percent yield, percent AE, PMI, and cumulative PMI obtained in Part 4.

\subsection{Part 6 - Energy consumption based on heating and cooling reaction solvents}

Since experimental procedures published in journal articles and patents are limited to disclosures of reaction temperature, pressure, and time when discussing reaction conditions with little to no mention of apparatuses used in unit operations and their physical and thermodynamic characteristics, onlycrude estimates of energy consumption for a given chemical reaction are possible. Furthermore, thermodynamic parameters such as heat capacities, heats of vaporization, etc. may not be available for reagents or advanced intermediates used in a pharmaceutical process. Hence, the focus of attention is on reaction and auxiliary solvents since most of the energy consumption in carrying out a reaction occurs during the reaction, workup, and purification operational phases, these solvents represent the bulk mass of ingredients in these stages, and physical and thermodynamic parameters for solvents are well documented in the literature [12]. Therefore, a table is given summarizing the cooling and heating energy consumptions in $\mathrm{kJ}$ for each reaction step based on cooling and heating the scaled masses of solvents from room temperature to the reaction or operational temperature needed to manufacture $1 \mathrm{~kg}$ of target product. Again, for easy visual display, a horizontal bar graph of results is given showing blue bars for cooling and red bars for heating contributions, respectively. Calculations of enthalpic energy contributions are based on employing heat capacity parameters for liquids and gases as necessary and the scaled number of moles of each solvent used leading to the production of $1 \mathrm{~kg}$ of target product as described previously [45]. 


\subsection{Part 7 - Environmental and safety-hazard impacts based on organic solvents}

When discussing environmental and safety-hazard impacts we have found that the literature on this subject under the umbrella term "life cycle assessment" is highly varied and complex with no standard of practice in place that is universally adopted. However, in our recent experience we have found that the Rowan solvent greenness index (RSGI) [46] is both very easy to use and thorough in its coverage of several kinds of impact parameters which makes it appealing to implement. The main attraction is that it is based on a given table of numbers which incorporates many impacts into single figures without having the user to search for contributing parameters separately in a myriad of print and online databases. Again, as in Part 6 the focus of attention is on all solvents used in a chemical reaction during the reaction, workup, and purification stages for the same reasons outlined above. In this analysis we have extended the original Rowan solvent greenness index (RSGI) to have a range between 0 and 12 instead of the original 0 to 10 as shown in Eq. 1:

$$
R S G I=\sum_{i} m_{i}\left(O S I_{12}\right)_{i}
$$

where $m_{i}$ is the mass of solvent $i(\mathrm{~kg})$ and the overall solvent index (OSI) normalized to be a number between 0 and 12 is given by Eq. 2:

$$
\left(O S I_{12}\right)_{i}=12\left(\frac{O S I_{i}-O S I_{\min }}{O S I_{\text {max }}-O S I_{\text {min }}}\right)
$$

where $O S I_{i}$ is the overall solvent index for solvent $i, O S I_{\min }$ is the minimum value of the overall solvent index over the entire set of solvents in the database, and $O S I_{\max }$ is the maximum value of the overall solvent index over the entire set of solvents in the database. In Eq. 1 the OSI values are non-arbitrary amplification factors of mass unlike the arbitrary Q-factors used in the original environmental quotient formalism [14]. For a given set of solvents a value of 0 for $O S I_{12}$ implies a comparatively benign solvent with minimal impact whereas a value of 12 implies a comparatively non-benign solvent with maximal impact. For a given solvent $i$, the overall solvent index is given by Eq. 3:

$$
\begin{aligned}
O S I_{i}= & 2\left(M_{O E L L, i}+M_{L D 50, i}+M_{L C 50, i}\right)+M_{G W P, i} \\
& +M_{S F P, i}+M_{O D P, i}+M_{A B P, i}+M_{B C P, i}+M_{P E R, i}+M_{\text {soil }, i} \\
& +M_{\text {half-life, } i}+M_{\text {aqua }, i}+M_{Q-\text { phrase }, i}+M_{S D, i}+M_{F P, i}
\end{aligned}
$$

where the metric parameters $(M)$ cover occupational exposure limit (OEL, ppm), LD50 (ingestion toxicity, mg/kg), LC50 (inhalation toxicity, $\mathrm{g} \mathrm{m}^{-3}$ for $4 \mathrm{~h}$ ), global warming potential (GWP, unitless), smog formation potential (SFP, unitless), ozone depletion potential (ODP, unitless), aciditybasicity potential (ABP, unitless), bioconcentration potential (BCP, unitless), persistence potential (PER, unitless), soil sorption coefficient (soil, $\mathrm{K}_{\mathrm{oc}}$ ), half-life of solvent in environment (half-life, h), aquatic toxicity to fish (aqua, $\mathrm{mg} / \mathrm{L}$ for $96 \mathrm{~h}$ ), Q-phrase potential (Q-phrase, unitless), skin dose (SD, mg), and flash point (FP, degrees K). In Eq. 3 in keeping with the original formalism [34], the OEL, LD50, and LC50 parameters are given twice the weight of the rest of the parameters since they are well recognized to have the most impact from an occupational safety and environmental point of view. For a given parameter $x$ for solvent $i$, the metric parameter $M$ is given by either of Eq. 4a-4c:

$$
\begin{aligned}
& M_{x, i}=1-\frac{\log x_{i}-\log x_{\text {min }}}{\log x_{\text {max }}-\log x_{\text {min }}} \\
& M_{x, i}=\frac{\log x_{i}-\log x_{\text {min }}}{\log x_{\text {max }}-\log x_{\text {min }}} \\
& M_{x, i}=0
\end{aligned}
$$

Equation $4 \mathrm{a}$ is used if the parameter $x_{i}$ increases with increasing greenness, Eq. $4 \mathrm{~b}$ is used if the parameter $x_{i}$ decreases with increasing greenness, and Eq. 4C is used if the parameter $x_{i}$ is unknown or equal to zero. For the acidity-basicity potential, absolute values are used for the $x_{i}$ parameters to avoid computational errors using the logarithm function in cases when negative numbers arise. The parameters applicable to Eq. $4 \mathrm{a}$ are: OEL, LD50, LC50, aquatic toxicity, and flash point where high values are consistent with benign conditions. The parameters applicable to Eq. 4b are: GWP, SFP, ODP, ABP, BCP, PER, soil adsorption, half-life, Q-phrase, and SD, where low values are consistent with benign conditions. It should be noted that expanding the list of solvents in a given database may result in changes to the maximum and minimum values for $O S I$ and $O S I_{12}$ and therefore to the rest of the OSI values for the intervening solvents depending on whether or not the additional solvents have higher or lower values than the existing maximum and minimum values. If the OSI values of the added solvents fall in between the existing limits then no changes to the magnitudes of the OSI values result in the rest of the solvents listed in the database. As the name suggests the Rowan solvent greenness analysis applies to all solvents used in a chemical process including reaction solvents, work-up solvents, and purification 
solvents. Generally, aqueous acidic and basic solvents are excluded since they are considered to be benign. The main focus is therefore on the impacts of organic solvents which generally comprise at least $75 \%$ of all materials used in a chemical manufacturing process. Furthermore, due to their frequent use in the chemical industry, toxicological and safety information for these substances are well described in the literature. Table 1 shows a list of $O S I_{12}$ values for organic solvents along with their respective colour ranges according to: green $\left(O S I_{12} \leq 5\right)$; yellow $\left(5<O S I_{12}<8\right)$; and red $\left(O S I_{12} \geq 8\right)$. In this compilation, the most benign solvent is water and the most impactful solvent is benzene. These OSI values are multiplied by the corresponding scaled masses of solvents used as reaction solvents, workup solvents, and purification solvents determined in the SYNTHESIS spreadsheet in order to obtain the overall RSGI score for the synthesis plan according to Eq. 1. A user does not have to consult any other data beyond the numbers given in Table 1 for the solvents used in a given chemical reaction which cover a wide range and are consistent with compilations of solvent guides in the literature [47-49].

\subsection{Part 8 - Cycle time process scheduling}

During a manufacturing process to a given pharmaceutical product, cycle or process times pertaining to each reaction step are important for scheduling purposes to minimize the time spent during all unit operations. The process time for a single reaction step is composed of reaction time, workup time, and purification time. Having this information in hand for all reaction steps in a synthesis allows for the construction of a Gantt scheduling diagram [50-53] which streamlines the unit operation flow from start to finish. Such a diagram has high utility for convergent plans in co-ordinating the start of convergent branches so that the intermediate prepared at the terminus of the convergent branch is ready at the same time as the intermediate prepared along the main branch so they are both utilized in the following convergent step with no time delay. A Gantt diagram also is highly useful in comparing process times for batch versus continuous flow operations. From the process times, other metrics relevant to the manufacturing process can also be obtained such as space-time-yield (STY, $\mathrm{kg} / \mathrm{m}^{3} / \mathrm{h}$ or $\mathrm{kg} / \mathrm{L} / \mathrm{h}$ ) defined as the ratio of mass of target product $(\mathrm{kg})$ to the product of total process time (h) times total volume of input materials $\left(\mathrm{L} \mathrm{or}^{3}\right.$ ) and volume time output (VTO) [54] defined on a per reaction step basis as shown in Eq. 5:

$$
V T O=\frac{t \times \sum V_{\text {reactors }}}{m_{\text {product }}}
$$

Table 1: Summary of overall solvent index (OSI) for various organic solvents used in the pharmaceutical industry.

\begin{tabular}{|c|c|}
\hline$O S I_{12}{ }^{a}$ & Solvent \\
\hline 7.545 & acetic acid \\
\hline 8.001 & acetic anhydride \\
\hline 5.867 & acetone \\
\hline 6.818 & acetonitrile \\
\hline 5.212 & amyl acetate \\
\hline 5.105 & amyl-t alcohol \\
\hline 5.149 & anisole \\
\hline 12.000 & benzene \\
\hline 5.594 & butanol-n \\
\hline 5.157 & butanol-sec \\
\hline 7.066 & butanol-tert \\
\hline 7.173 & carbon disulfide \\
\hline 8.755 & carbon tetrachloride \\
\hline 10.320 & chlorobenzene \\
\hline 8.388 & chloroform \\
\hline 7.975 & cyclohexane \\
\hline 5.068 & cyclopentanone \\
\hline 7.341 & cyclopentyl methyl ether \\
\hline 9.505 & dichlorobenzene-1,2 \\
\hline 9.456 & dichloroethane-1,2 \\
\hline 7.060 & dichloromethane \\
\hline 7.335 & diethyl ether \\
\hline 3.553 & diglyme \\
\hline 3.456 & dimethyl carbonate \\
\hline 2.199 & dimethyl sulfoxide \\
\hline 8.184 & dimethylacetamide \\
\hline 6.861 & dimethylformamide \\
\hline 1.575 & dimethylisosorbide \\
\hline 7.073 & dioxane-1,4 \\
\hline 3.139 & ethanol \\
\hline 6.647 & ethyl acetate \\
\hline 4.227 & ethylene glycol \\
\hline 2.699 & ethylene glycol dimethyl ether \\
\hline 5.113 & ethylene glycol monomethyl ether \\
\hline 8.582 & formaldehyde \\
\hline 3.808 & glycol diacetate \\
\hline 6.203 & heptane-n \\
\hline 6.210 & heptanol-1 \\
\hline 7.551 & hexamethylphosphoric triamide \\
\hline 8.073 & hexane-n \\
\hline 5.171 & isoamyl acetate \\
\hline 5.376 & isoamyl alcohol \\
\hline
\end{tabular}

(Continued) 
Table 1: Continued

\begin{tabular}{|c|c|}
\hline$O S I_{12}{ }^{a}$ & Solvent \\
\hline 4.833 & isobutyl acetate \\
\hline 4.075 & isooctane \\
\hline 5.486 & isopropanol \\
\hline 6.583 & isopropyl acetate \\
\hline 4.471 & isopropyl ether \\
\hline 3.950 & methanol \\
\hline 5.486 & methyl acetate \\
\hline 6.266 & methyl ethyl ketone \\
\hline 5.735 & methyl formate \\
\hline 5.994 & methyl propionate \\
\hline 6.512 & methyl t-butyl ether \\
\hline 7.928 & methylcyclohexane \\
\hline 8.175 & methyl tetrahydrofuran-2 \\
\hline 10.319 & nitrobenzene \\
\hline 6.393 & nitromethane \\
\hline 5.124 & $\mathrm{~N}$-methylpyrrolidinone \\
\hline 4.871 & octanol-1 \\
\hline 4.886 & pentane-n \\
\hline 6.856 & petroleum ether \\
\hline 5.679 & propanol-1 \\
\hline 2.346 & propylene carbonate \\
\hline 1.570 & propylene glycol \\
\hline 9.791 & pyridine \\
\hline 3.336 & sulfolane \\
\hline 7.235 & tetrahydrofuran \\
\hline 5.180 & thionyl chloride \\
\hline 9.627 & toluene \\
\hline 6.672 & trichloroethylene \\
\hline 9.776 & triethylamine \\
\hline 2.736 & triethylene glycol monomethyl ether \\
\hline 5.360 & trifluoroacetic acid \\
\hline 6.657 & trifluorotoluene \\
\hline 0.000 & water \\
\hline 9.156 & xylene-m \\
\hline 9.288 & xylene-o \\
\hline 9.333 & xylene-p \\
\hline
\end{tabular}

a Green colour indicates benign performance $\left(O S I_{12} \leq 5\right)$; yellow colour indicates intermediate performance $\left(5<O S I_{12}<8\right)$; and red colour indicates worst performance $\left(\mathrm{OSI}_{12} \geq 8\right)$.

where $t$ is the time per batch (h); $V_{\text {reactors }}$ is the nominal volume of all reactors $\left(\mathrm{m}^{3}\right)$; and $m_{\text {product }}$ is the mass of output product $(\mathrm{kg})$. Since only reaction times are typically given in literature experimental procedures, it is not possible to estimate proper cycle or process times for each reaction step or for an entire synthesis plan, and thus it is not possible to construct Gantt scheduling diagrams for a given manufacturing chemical process. Furthermore, such details about a manufacturing process are proprietary since they are very closely linked to various costs of production. Hence, Part 8 is omitted in the three illustrative process green synthesis reports shown in this work. However, we illustrate how a Gantt diagram may look like for a hypothetical continuous manufacturing process in Figure 4. Reaction, workup, and purification times for each step are colour coded as red, white, and blue, respectively. In this example, the synthesis plan is composed of three branches and two convergent steps. The main branch, $B_{1}$, is composed of 5 steps covering a 41-hour period, the second branch $\mathrm{B}_{2}$ is composed of 2 steps covering a 16-hour period, and the third branch $\mathrm{B}_{3}$ is composed of one step covering an 8-hour period. We note that in order for the second intermediate in branch $\mathrm{B}_{2}$ to be ready at hour 25 , the second branch needs to be initiated 9 hour after the start of the main branch $\mathrm{B}_{1}$. Similarly, the third branch needs to be initiated 24 hour after the main branch $\mathrm{B}_{1}$ begins in order for its terminal intermediate to be ready at hour 32 coinciding with the fourth intermediate collected along the main branch. Purification times occur only in steps leading up to the key convergent steps and for the final reaction step in the synthesis.

\section{Illustrative examples of process green synthesis reports}

In this section we highlight the main findings of the respective reports for the following pharmaceuticals: 5-HT2B and 5-HT7 receptors antagonist, brivanib, and orexin receptor agonist. The full reports are given in the Supplementary material along with a blank template that can be used for any synthesis plan.

\subsection{Example 1: 5-HT2B and 5-HT7 receptors antagonist}

The synthesis plan is linear consisting of 5 reaction steps and involves a classical resolution using brucine and cinchonidine diastereomeric salts to obtain the desired enantiomeric product in step 4. The most material efficient step with the lowest PMI is step 3 which involves an intramolecular [5+1] cyclization; whereas, the least material efficient step with the highest PMI is the last step which involves a tandem amidation-hydrochloride 


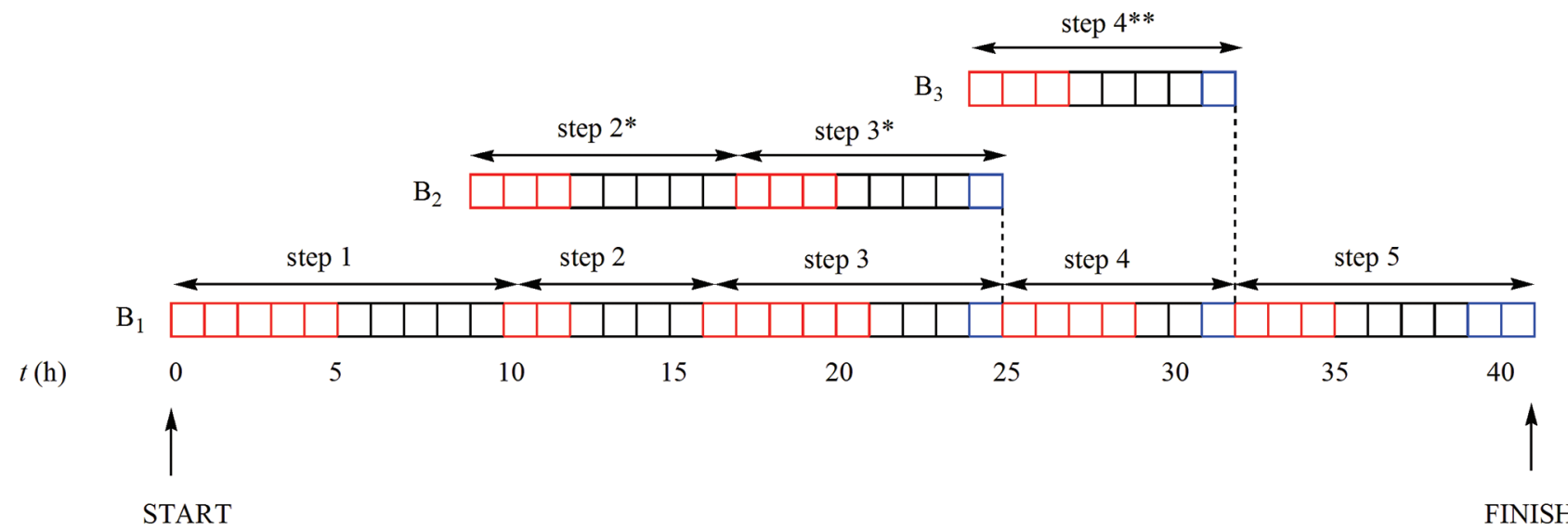

Figure 4: Example Gantt diagram for a hypothetical multi-convergent synthesis having three branches: $B_{1}$ ( 5 steps), $B_{2}(2$ steps), and $B_{3}$ (1 step).

salt formation sequence due to the high workup solvent consumption. Steps 3 and 4 had the highest atom economies; whereas, steps 2 (tandem reductionhydrolysis) and 5 (tandem amidation-hydrochloride salt formation) had the worst. About $54 \%$ of the mass of reagents used in this synthesis plan is sacrificial mainly arising from the methanol used in the potassium formate reduction reaction in step 2 . The mass process block diagram indicates that the second and last steps produce the most organic solvent waste at 189 and $151 \mathrm{~kg}$ per $\mathrm{kg}$ of target product, respectively. The second step produces the most by-product waste at $3.45 \mathrm{~kg}$ per $\mathrm{kg}$ of target product. The second and fourth steps require the most heating requirements pertaining to the tandem reductionhydrolysis and classical resolution steps, carried out at 35 and $60^{\circ} \mathrm{C}$, respectively. The first step requires the most cooling requirements pertaining to the step involving the addition of an acetylide to the ketone substrate carried out at $-25^{\circ} \mathrm{C}$. The greenest reaction solvent used is diglyme in step 3; whereas the most impactful solvent used is toluene in steps 2 and 1 in the workup and purification phases, respectively. The workup phases for all steps produces by far the largest RSGI score contribution.

\subsection{Example 2: brivanib}

The synthesis plan is convergent involving two branches consisting of 3 reactions in the second branch for a total of 15 reaction steps. The most material efficient steps with the least PMI are steps 4 and 12 involving a [4+2] cycloaddition with formamide and hydrogenation, respectively. The least material efficient step with the highest PMI is step 3 involving amination using diphenyl phosphoryl hydroxylamine reagent. This is due to the high E-workup contribution to overall waste produced. Steps 11 (esterification with benzyloxycarbonyl protected D-alanine and EDAC) and 12 had the best atom economies; whereas, steps $1([3+2]$ cycloaddition) and $6 \mathrm{~b}$ (tandem alkylation-decarboxylation-aromatic substitution) had the worst. About $63 \%$ of the mass of reagents used in this synthesis plan is sacrificial owing to esterification protection, sacrificial chlorine substitution, esterification using EDAC as a sacrificial reagent to mop up the water by-product, demethylation, and reduction reactions in steps $7,8,11,7 \mathrm{~b}$, and $8 \mathrm{~b}$, respectively. The mass process block diagram indicates that step 3 produces the most organic waste at $1257 \mathrm{~kg}$ per $\mathrm{kg}$ of target product. The first step involving a [3+2] cycloaddition between ethyl crotonate and tosylmethyl isocyanide produces the most by-product waste at $4.86 \mathrm{~kg}$ per $\mathrm{kg}$ of target product. Step 1 has the highest cooling requirements pertaining to a reaction temperature of $-78^{\circ} \mathrm{C}$, and step 4 has the highest heating requirements pertaining to a reaction temperature of $165^{\circ} \mathrm{C}$ for the respective reaction solvents. The most impactful reaction solvent, workup solvent, and purification solvent is dimethylformamide used in step 3, ethyl acetate used in step 3, and methyl $t$-butyl ether used in step $6 \mathrm{~b}$, respectively. The workup phases for all steps produces by far the largest RSGI score contribution.

\subsection{Example 3: orexin receptor agonist}

The synthesis plan is convergent involving two branches consisting of 4 reactions in the second branch for a total of 12 reaction steps. The unique features of this synthesis are a tandem biotransamination-[6+0] cyclization reaction in step 2 using D-alanine and D-glucose leading to a diastereomeric piperidone intermediate, followed by a 
tandem reduction-classical resolution sequence using (+)-10-camphorylsulfonic acid as the resolving agent in step 4. The most material efficient step with the least PMI is step 1 involving base catalyzed alkylation of dimethyl malonate with methyl vinyl ketone; whereas, the least material efficient step with the highest PMI is step 6 involving an etherification reaction with 5-fluoro-1-hydroxypyridine and a tosylate intermediate. Again, the high E-reaction solvent and E-workup contributions were the source of the high PMI value of 153 for step 6. Steps 1 and $4 \mathrm{~b}$ (esterification of 2-iodo5-methylbenzoic acid) had the highest atom economies; whereas, steps 2, 5 (tandem N-Boc amino protectiontosylation), 6, and 6b (Suzuki coupling) had the lowest atom economies. About $89 \%$ of the mass of reagents used in this synthesis plan is sacrificial owing to sacrificial D-glucose used to convert pyruvic acid by-product to L-lactic acid in step 2, tandem N-Boc protection-tosylation in step 5, N-Boc deprotection in step 7, high methanol consumption in the esterification reaction of step $4 \mathrm{~b}$, and sacrificial aromatic substitution in step $5 \mathrm{~b}$ using pinacolborane. The mass process block diagram indicates that the resolution step 4 produces the most organic solvent waste at $88.5 \mathrm{~kg}$ per $\mathrm{kg}$ of target product and that the etherification reaction of step 6 produces the most aqueous solvent waste at $114.8 \mathrm{~kg}$ per $\mathrm{kg}$ of target product. Step 4 has the highest cooling requirement pertaining to a reaction temperature of $-25^{\circ} \mathrm{C}$ for the lithium aluminum hydride reduction and convergent step 8 has the highest heating requirement pertaining to a reaction temperature of $44^{\circ} \mathrm{C}$ for the final amidation reaction. The most impactful reaction solvent, workup solvent, and purification solvent is tetrahydrofuran in step 4, ethyl acetate in step 2, and heptane in step 8, respectively; albeit step 8 is the only one that utilized purification solvents. Again, the workup phases for all steps produces by far the largest RSGI score contribution.

\section{Ranking synthesis plans to a common target product}

The problem of ranking synthesis plans according to a set of attributes in a reliable and unbiased way is an important one particularly when comparing the overall performances of plans to a common target product. We illustrate how this can be done using data given in Table 2 for the following metrics for 6 industrial plans to the pharmaceutical apixaban: PMI, mass of sacrificial reagents (SR) per kg of apixaban, input enthalpic energy (IEE) for solvents (kJ per kg apixaban), and RSGI(overall) for all organic solvents used.
Table 2: Summary of essential metrics for 6 industrial plans to apixaban.

\begin{tabular}{lcccc}
\hline Plan & PMI & $\begin{array}{c}\text { SR (kg/kg } \\
\text { product) }\end{array}$ & $\begin{array}{c}\text { IEE } \\
\text { (heating and cooling, } \\
\text { kJ/kg product) }\end{array}$ & $\begin{array}{c}\text { RSGI } \\
\text { (overall, } \mathbf{~ k g})\end{array}$ \\
\hline BMS 2003 & 626 & 27.67 & 8562 & 2660 \\
BMS 2006 & 245 & 12.43 & 13254 & 626 \\
DrReddy & 879 & 35.56 & 58213 & 3881 \\
Jiang-Ji & 383 & 24.33 & 6820 & 1180 \\
MSN & 498 & 36.26 & 20771 & 1855 \\
Optimus & 379 & 24.01 & 16610 & 1327 \\
\hline
\end{tabular}

The three ranking methods examined here are vector magnitude ratio (VMR) [12], poset method [31], and Borda positional count method [32,33]. Based on the 4 attributes listed in Table 2, the VMR method is given by Eq. 6:

$$
\begin{aligned}
V M R & =\frac{\sqrt{(P M I)^{2}+(S R)^{2}+(I E E)^{2}+(R S G I)^{2}}}{\sqrt{\left((P M I)_{\min }\right)^{2}+\left((S R)_{\text {min }}\right)^{2}+\left((I E E)_{\text {min }}\right)^{2}+\left((R S G I)_{\text {min }}\right)^{2}}} \\
& =\sqrt{(P M I)^{2}+(S R)^{2}+(I E E)^{2}+(R S G I)^{2}}
\end{aligned}
$$

where the optimal attribute metrics are given by $(\mathrm{PMI})_{\text {min }}=1$, $(\mathrm{SR})_{\min }=0,(\mathrm{IEE})_{\min }=0$, and (RSGI) ${ }_{\text {min }}=0$. A plan is ranked high if its VMR value is low since low values of each of the four attributes contribute to an overall low value of VMR. The ranking order based on VMR values is as follows: Jiang-Ji (6932), BMS 2003 (8988), BMS 2006 (13271), Optimus (16667), MSN (20860), and DrReddy (58349). The VMR analysis suggests that the best performing plan for apixaban is the Jiang-Ji plan.

The poset ranking method is based on determining all pairwise comparisons of plans according to all pairwise attributes and calculating the number of occurrences of dominances of each plan. Since there are 6 synthesis plans there are 15 possible pairwise plan comparisons, and since there are 4 attributes there are 6 possible pairwise attribute comparisons for each pairwise plan comparison. This means that a poset analysis on these data will require a total of 15 X $6=90$ pairwise comparisons. In general, a complete poset analysis on $K$ synthesis plans to a common target product based on $m$ attributes will require $\mathrm{C}(K, 2)^{\star} \mathrm{C}(m, 2)=\left(K ! /((K-2) ! 2 !)^{\star}(m ! /(m-2) ! 2 !)\right.$ pairwise comparisons. Hence, in this example, for each type of pairwise attribute comparison, an upper triangular 6 by 6 matrix is constructed and the number of comparable and incomparable pairs is determined. A comparable pair is 
defined as a case where plan A performs better or worse than plan B for both attributes C and D. An incomparable pair is defined as a case where plan A performs better (worse) than plan B for attribute $\mathrm{C}$, but plan B performs better (worse) than plan A for attribute D. When a comparable pair for a given pairwise attribute comparison is found the dominant plan is identified. This sequence of steps is repeated for each pairwise attribute comparison and then the number of dominant occurrences for each plan are tallied up. As an example, if we examine the PMI versus input energy attributes in Table 2 we find the following respective ranking orders: BMS $2006>$ Optimus $>$ Jiang-Ji > MSN > BMS 2003 > DrReddy for PMI and Jiang-Ji $>$ BMS $2003>$ BMS $2006>$ Optimus > MSN > DrReddy for input enthalpic energy. We find 10 occurrences of comparable and 5 occurrences of incomparable pairs. Among the 10 comparable pair instances, we count the following number of occurrences of dominance: BMS 2006 (3), Optimus (2), Jiang-Ji (3), MSN (1), BMS 2003 (1), and DrReddy (0). Repeating this procedure for the remaining 5 pairwise attribute comparisons (PMI versus SR, PMI versus RSGI, SR versus IEE, SR versus RSGI, and IEE versus RSGI) we finally end up with the following overall number of occurrences of dominance for each plan ranked in descending order: BMS 2006 (24), Jiang-Ji (19), Optimus (16), BMS 2003 (7), MSN (4), and DrReddy (0). The Supplementary material contains a full analysis of the poset analysis applied to the 6 apixaban plans. From the poset analysis we conclude that this is the overall ranking order of the 6 plans based on the 4 attributes where the BMS 2006 plan is ranked highest.

In the Borda count method we first rank the set of plans according to a given attribute. Since a low value for each attribute listed in Table 2 implies best performance and since there are 6 plans, we assign a rank value of 1 for the plan having the lowest value for a given attribute and a rank value of 6 for the plan having the highest value for a the same attribute. For a given rank order for a given attribute, we assign points to each plan starting with 6 for the top ranked plan all the way to 1 for the least ranked plan. In general, if we have $K$ plans then the number of points assigned to a given plan is equal to $K+1-r$, where $r$ is the rank number based on a given attribute. We repeat the process of ranking and point assignments for each attribute to obtain the overall point score for each plan according to Eq. 7.

$$
\begin{aligned}
\text { points }_{\text {overall }} & =\text { points }_{P M I}+\text { points }_{S R}+\text { points }_{I E E}+\text { points }_{R S G I} \\
& =K+1-r_{P M I}+K+1-r_{S R}+K+1-r_{I E E}+K+1-r_{R S G I} \\
& =4(K+1)-r_{P M I}-r_{S R}-r_{I E E}-r_{R S G I}
\end{aligned}
$$

The maximum number of overall points achievable is $4 K$ (all the $r$ values are equal to 1) and the minimum number of overall points achievable is 4 (all the $r$ values are equal to $K$ ). The Borda count procedure leads to the following point score in descending order: BMS 2006 (22), Jiang-Ji (19), Optimus (17), BMS 2003 (12), MSN (9), and DrReddy (5) suggesting that the BMS 2006 plan is ranked highest. The Supplementary material contains a full Borda count analysis of the 6 apixaban plans. This rank order is identical to the one determined by the poset method but is different from the one determined by the VMR method.

Restrepo and Stadler [31] point out that the VMR method suffers from a priori aggregation or weighting of attributes, such as the root-mean-square function in Eq. 6, and that pairwise attribute comparisons are hidden in a single number, and hence advocate for a ranking method based on an "order theoretic approach" to circumvent these issues. This statement is true if the set of attributes considered in the VMR analysis trend in different optimal directions, that is, if some attributes in the set are ranked high if their values are low and other attributes are ranked high if their values are also high. Therefore, A VMR analysis on mixed trend attributes would not be reliable. However, when all attributes in the set trend in the same optimal direction, as in the case of PMI, SR, IEE, and RSGI all having low values for optimality, these problems are not expected to be an issue for the VMR method. The strength of the poset and Borda count methods is that they can handle mixed trend attributes in the ranking procedures; whereas, the VMR method cannot. The different rank order outcome found in the apixaban example by the VMR analysis compared to either the poset or Borda methods is attributable to the unbounded nature of the 4 attributes. There exist only lower bounds for these attributes as indicated in Eq. 6. However, each of these attributes does not have an accompanying upper bound, which results in a numerical magnitude problem with respect to ranking VMR values. We have found that the Borda count procedure applied to the 4 attributes also avoids the problems of the VMR approach, as does the poset method, but it leads to much simpler and faster computations than the poset method, particularly when the number of plans to a given target molecule and the number of attributes considered are both large. Moreover, we also find that the Borda method, which also has built into it pairwise attribute comparisons, reproduces a ranking order identical to that found by the poset method. As a check, we re-examined the test example of 4 different reactions to produce 3-benzyl1,3-oxazin-2-one cited in Restrepo and Stadler's work [55] by the poset and Borda count methods described in this work using the 5 attributes of atom economy, reaction 
yield, inverse of stoichiometric factor, material recovery parameter, and reaction mass efficiency. Full details are presented in the Supplementary material. In this second example we also found identical ranking orders for the 4 reactions by the poset and Borda methods; namely $\mathrm{r} 4>\mathrm{r} 1$ $>\mathrm{r} 2>\mathrm{r}$ 3. We also did a VMR analysis according to Eq. 8 and found that the rank order was also $r 4>r 1>r 2>r 3$.

$$
\begin{aligned}
V M R & =\frac{\sqrt{(A E)^{2}+(Y)^{2}+(1 / S F)^{2}+(M R P)^{2}+(R M E)^{2}}}{\sqrt{\left((A E)_{\text {max }}\right)^{2}+\left((Y)_{\text {max }}\right)^{2}+\left((1 / S F)_{\text {max }}\right)^{2}}} \\
& =\frac{\sqrt{(A E)^{2}+(Y)^{2}+(1 / S F)^{2}+(M R P)^{2}+(R M E)^{2}}}{\sqrt{5}}
\end{aligned}
$$

where the optimal attribute metrics are given by $(\mathrm{AE})_{\max }=1$, $(\mathrm{Y})_{\text {max }}=1,(1 / \mathrm{SF})_{\text {max }}=1,(\mathrm{MRP})_{\text {max }}=1$, and $(\mathrm{RME})_{\text {max }}=1$. A reaction is ranked high if its VMR value approaches 1 since high values approaching 1 for each of the 5 attributes contribute to an overall high value of VMR. In this case all three ranking methods yielded the same result. This outcome with respect to the VMR method is likely due to the fact that the 5 attributes all range between 0 and 1 , with a value of 1 indicating optimality, i.e. high rank. Since the 5 attributes trend in the same direction with respect to ranking and also have both lower and upper bounds, then the resulting VMR ranking order is expected to be the same, or nearly the same, as the Borda or poset methods. There should be at least a matching of the highest and lowest ranked reactions in all three methods, but some variation may be observed in the ranking of the "in between" reactions from the VMR analysis. Hence, we can conclude that the more the comparative attributes have unbounded values and trend in different directions, the more likely it is expected that a VMR analysis will yield a different ranking order than the poset or Borda methods. Such a VMR ranking order would necessarily be considered unreliable. Based on the findings presented in this work we suggest that the poset and Borda ranking methods should result in very good agreement in the ranking orders.

We also point out that though a highest performing plan can be identified among a set of plans, there remains room for further improvement with respect to the attributes considered if the number of occurrences of dominance or the Borda count point score is less than the ideal maximum values of $(K-1)^{\star}$ (number of pairwise attribute comparisons) and $K^{\star}$ (number of attributes), respectively where $K$ is the number of synthesis plans considered. In the case of the apixaban example with 6 plans, 4 attributes, and 6 pairwise attribute comparisons, the maximum number of dominances possible is $(6-1)^{\star} 6=30$ and the maximum Borda count score is $6^{\star} 4=24$. The highest ranking BMS 2006 plan had 24 out of 30 possible occurrences of dominance and a Borda count of 22 out of a possible 24 indicating that further improvements could be achieved in exactly those instances when incomparable attribute occurrences were found in the poset analysis or when the plan rank number is greater than 1 for a given attribute. In the case of the BMS 2006 plan it had a top ranking in all attributes except the input enthalpic energy where it ranked in third place. Hence, this is an identifiable area for further optimization for this plan. If a plan achieves the highest dominance and Borda count scores across all attributes, then it can be declared universally optimal among a set of competing plans. The list of 4 attributes considered in both types of analysis can, of course, be extended to include more attributes such as process time and cost if these proprietary data are available. With more attributes included in the analysis, it is obvious that the ranking order will necessarily change; however, the ultimate goal of achieving top ranking among all attributes remains the same, though it will be a much more challenging task.

\section{Conclusions}

We have described a comprehensive yet very easy to implement protocol for the contents of a process green chemistry report covering material efficiency, energy efficiency, and environmental and safety-hazard impact. Such a report communicates succinctly the essential features by which a given synthesis plan can be judged according to green chemistry principles, thereby directing decision making so that further improvements can be made. It also provides a comprehensive evaluation of "greenness" of a given chemical process that goes beyond the customary declarations in the literature based on the performance of one or two variables typically favouring material efficiency only such as number of reaction steps, overall yield, atom economy, or process mass intensity. Due to the availability and reliability of toxicological, environmental, and thermodynamic data for solvents [9], and their dominant mass contribution to all materials used in a chemical process, these substances are the main focus of attention in the assessment of environmental and safety-hazard impact and energy consumption in Parts 6 and 7 of the process green synthesis report. Gantt scheduling diagrams can be included in the report when complete process times are available for the reaction, 
workup, and purification phases for each reaction step. We have illustrated the construction of process green synthesis reports for three syntheses of APIs currently implemented in the pharmaceutical industry. Information contained in comparative process green chemistry reports of competing synthesis plans to the same target product are expected to be extremely useful in helping to direct further optimizations. In this regard, ranking of such synthesis plans in a reliable and unbiased way is a key issue. We have found that the Borda count method is satisfactory for achieving this goal; it is easy and fast to compute compared to the poset method and it avoids the problems associated with the VMR method.

Acknowledgements: Marco Eissen is thanked for useful discussions.

Supplementary material: Contains process green synthesis reports for 5-HT2B and 5-HT7 receptors antagonist, brivanib, and orexin receptor agonist pharmaceuticals, a blank template report that can be used for any synthesis plan; poset and Borda count analyses for 6 apixaban synthesis plans and 4 reactions producing 3-benzyl-1,3-oxazin-2-one.

\section{References}

[1] Anderson N.G., Practical Process Research and Development a guide for organic chemists (2nd ed.). Elsevier, Amsterdam, 2012.

[2] Harrington P.J., Pharmaceutical Process Chemistry for Synthesis rethinking the routes to scale-up. Wiley, New Jersey, 2011.

[3] Koenig S.G. (Ed.), Scalable Green Chemistry - case studies from the pharmaceutical industry. CRC Press-Taylor \& Francis, Boca Raton, 2013.

[4] Peterson E.A., Manley J.B., (Eds.), Green Chemistry Strategies for Drug Discovery. Royal Society of Chemistry, London, 2015.

[5] Dunn P.J., Hii K.K., Krische M.J., Williams M.T., Sustainable Catalysis - challenges and practices for the pharmaceutical and fine chemical industries. Wiley, New Jersey, 2013.

[6] Xian M., Sustainable Production of Bulk Chemicals - integration of bio-, chemo-resources and processes. Springer, Dordrecht, 2016.

[7] Dunn P.J., Wells A.S., Williams M.T. (Eds.), Green Chemistry in the Pharmaceutical Industry. Wiley-VCH, Weinheim, 2010.

[8] Yasuda N. (Ed.), The Art of Process Chemistry. Wiley-VCH, Weinheim, 2011.

[9] Hessel V., Kralisch D., Kockmann N., Novel Process Windows Innovative Gates to Intensified and Sustainable Chemical Processes. Wiley-VCH, Weinheim, 2015.
[10] Boodhoo K., Harvey A. (Eds.), Process Intensification for Green Chemistry - Engineering Solutions for Sustainable Chemical Processing. Wiley, Chichester, 2013.

[11] Jiménez-González C., Constable D.J.C., Green Chemistry and Engineering - A Practical Design Approach. Wiley, Hoboken, New Jersey, 2011.

[12] Andraos J., Useful Tools for the Next Quarter Century of Green Chemistry Practice: A Dictionary of Terms and a Data Set of Parameters for High Value Industrial Commodity Chemicals. ACS Sustain. Chem. Eng., 2018, 6, 3206-3214.

[13] Roschangar F., Sheldon R., Senanayake C.H., Overcoming barriers to green chemistry in the pharmaceutical industry - the Green Aspiration Level concept. Green Chem., 2015, 17, 752-768.

[14] Sheldon R.A., Consider the Environmental Quotient. ChemTech, 1994, 24(3), 38-47.

[15] Jiménez-González C., Ponder C.S., Broxterman Q.B., Manley J.B., Using the right green yardstick: why process mass intensity is used in the pharmaceutical industry to drive more sustainable processes. Org. Process Res. Dev., 2011, 15, 912-917.

[16] Ribeiro, M.G.T.C., Costa D.A., Machado A.A.S.C., Greenness of chemical reactions - limitations of mass metrics. Green Chem. Lett. Rev., 2013, 6, 1-18.

[17] Edwards D.W., Lawrence D., Assessing the inherent safety of chemical process routes: is there a relation between plant costs and inherent safety? Process Saf. Environ., 1993, 71, 252-258.

[18] Eissen M. Bewertung der Umweltverträglichkeit organischchemischer Synthesen. PhD thesis, Universität Oldenburg, Germany, 2001.

[19] Eissen M., Metzger J.O., Environmental Performance Metrics for Daily Use in Synthetic Chemistry. Chem. Eur. J., 2002, 8, 35803585.

[20] EATOS - Environmental Assessment Tool for Organic Syntheses, www.metzger.chemie.uni-oldenburg.de/eatos/english.htm.

[21] Van Aken K., Strekowski L., Patiny L., EcoScale, a semiquantitative tool to select an organic preparation based on economical and ecological parameters. Beilstein J. Org. Chem., 2006, 2(3), DOI:10.1186/1860-5397-2-3.

[22] Ribeiro M.G.T.C., Costa D.A., Machado A.A.S.C., "Green Star": a holistic Green Chemistry metric for evaluation of teaching laboratory experiments. Green Chem. Lett. Rev., 2010, 3, 149-159.

[23] Curzons A.D., Jiménez-González C., Duncan A.L., Constable D.J.C., Cunningham V.L., Fast life cycle assessment of synthetic chemistry (FLASC ${ }^{\mathrm{TM}}$ ) tool. Int. J. Life Cycle Ass., 2007, 12, 272-280.

[24] PMI Predictor, https://acsgcipr-predictpmi.shinyapps.io/pmi_ calculator/_w_3597011e/.

[25] Yoshida S., Kasai M., Kimura T., Akiba T., Takahashi T., Sakamoto S., Development of a Practical and Scalable Synthesis of a Potent Selective Dual Antagonist for 5-HT2B and 5-HT7 Receptors. Org. Process Res. Dev., 2012, 16, 654-663. 
[26] Pesti J.A., LaPorte T., Thornton J.E., Spangler L., Buono F., Crispino G., et. al., Commercial Synthesis of a PyrrolotriazineFluoroindole Intermediate to Brivanib Alaninate: Process Development Directed toward Impurity Control. Org. Process Res. Dev., 2014, 18, 89-103.

[27] Lobben P.C., Barlow E., Bergum J.S., Braem A., Chang S.Y., Gibson F., et. al., Control Strategy for the Manufacture of Brivanib Alaninate, a Novel Pyrrolotriazine VEGFR/FGFR Inhibitor. Org. Process Res. Dev., 2015, 19, 900-908.

[28] Hunt J.T., Bhide R.S., Borzilleri R.M., Qian L., Pyrrolotriazine Inhibitors and Kinases. WO 0071129 (Bristol-Myers Squibb Co., 2000).

[29] Crispino G.A., Hamedi M., LaPorte T.L., Thornton J.E., Pesti J.A., Xu Z., et. al., Process for the Preparation of [(1R),2S]-2Aminopropionic acid 2-[4-(4-fluoro-2-methyl-1H-indol-5-yloxy)5-methylpyrrolo[2,1-F][1,2,4]triazin-6-yloxy]-1-methylethyl ester. US2007249610 (Bristol-Myers Squibb Co., 2007).

[30] Girardin M., Ouellet S.G., Gauvreau D., Moore J.C., Hughes G., Devine P.N., et. al., Convergent Kilogram-Scale Synthesis of Dual Orexin Receptor Antagonist. Org. Process Res. Dev., 2012, 17, 61-69.

[31] Restrepo G., Stadler P.F., Assessing greenness of chemical reactions and synthesis plans through poset-valued landscapes. ACS Sustain. Chem. Eng., 2016, 4, 2191-2200.

[32] Levin J., Nalebuff B., An Introduction to Vote Counting Schemes. J. Econ. Perspect., 1995, 9, 3-26.

[33] Saari D.G. The Optimal Ranking Method Is the Borda Count. International Institute for Applied Systems Analysis, Laxenburg, Austria, 1985.

[34] Zhou J., Oh L.M., Ma P., Li H.Y., Confalone P., Synthesis of 4,5-Dihydro-pyrazolo-[3,4c]-pyrid-2-ones. US2003181466 (BristolMyers Squibb Company, 2003).

[35] Shapiro R., Rossano L.T., Mudryk B.M., Cuniere N., Obelholzer M., Zhang H., et al., Process for Preparing 4,5-Dihydro-pyrazolo[3,4c]-pyrid-2-ones. US2006069258 (Bristol-Myers Squibb Company, 2006).

[36] Jiang J., Ji Y., Alternative Synthesis of Apixaban (BMS-562247), an Inhibitor of Blood Coagulation Factor XA. Synth. Commun., 2013, 43, 72-79.

[37] Rajan S.T., Eswaraiah S., Venkatesh M., Novel Intermediate and Polymorphs of 1-(4-Methoxyphenyl)-7-oxo-6-[4-(2-oxopiperidin1-yl)phenyl]-4,5,6,7-tetrahydro-1H-pyrazolo-[3,4c]-pyridine3-carboxamide and process thereof. US2015353541 (MSN Laboratories Limited, 2015).

[38] Nevuluri N.R., Rapolu R.K, Iqbal J., Kandagatla B., Sen S., Dahanukar V.H., et al., A morpholine-free process amenable convergent synthesis of apixaban: a potent factor Xa inhibitor. Monatsh. Chem., 2017, 148, 1477-1482.

[39] Reddy D., Reddy S., Rane D.R., Velivela S.R., An Improved Process for the Preparation of Apixaban. W02017187245 (Optimus Drugs (P) Limited, 2017).
[40] Andraos J., On Using Tree Analysis to Quantify the Material, Input Energy, and Cost Throughput Efficiencies of Simple and Complex Synthesis Plans and Networks: Towards a Blueprint for Quantitative Total Synthesis and Green Chemistry. Org. Process Res. Dev., 2006, 10, 212-240.

[41] Andraos J., Sayed M., On the Use of 'Green' Metrics in the Undergraduate Organic Chemistry Lecture and Laboratory to Assess the Mass Efficiency of Organic Reactions. J. Chem. Educ., 2007, 84, 1004-1011.

[42] Andraos J., Hent A., A Simplified Application of Material Efficiency Green Metrics to Synthesis Plans - Pedagogical Case Studies Selected From Organic Syntheses. J. Chem. Educ., 2015, 92, 1820-1830.

[43] Andraos J., Reaction Green Metrics: Problems, Exercises, and Solutions. CRC Press-Taylor \& Francis, Boca Raton, 2019.

[44] Andraos J., Relationships between step and cumulative PMI and E-factors: implications on estimating material efficiency with respect to charting synthesis optimization strategies. Green Process. Synth., 2019, 8, 324-336.

[45] Andraos J., Complete Green Metrics Evaluation of Various Routes to Methyl Methacrylate According to Material and Energy Consumptions and Environmental and Safety Impacts: Test Case from the Chemical Industry. ACS Sust. Chem. Eng., 2016, 4, 312-323.

[46] Slater C.S., Savelski M., A method to characterize the greenness of solvents used in pharmaceutical manufacture. J. Environ. Sci. Heal. A, 2007, 42, 1595-1605.

[47] Prat D., Hayler J., Wells A., A Survey of Solvent Selection Guides. Green Chem., 2014, 16, 4546-4552.

[48] Prat D., Wells A., Hayler J., Seddon H., McElroy C.R., AbouShehada S., et al., CHEM21 Selection Guide of Classical and Less Classical Solvents. Green Chem., 2016, 18, 288-297.

[49] Diorazio L.J., Hose D.R.J., Adlington N.K., Towards a More Holistic Framework for Solvent Selection. Org. Process Res. Dev., 2016, 20,760-773.

[50] Gantt H.L., A graphical daily balance in manufacture. T. Am. Soc. Mech. Eng., 1903, 24, 1322-1336.

[51] Gantt H.L., Organizing for Work. Harcourt, Brace, and Howe, New York, 1919.

[52] Ku H., Rajagopalan D., Karimi I., Scheduling in batch 596 processes. Chem. Eng. Prog., 1987, 83(8), 35-46.

[53] Musier R.F.H., Evans L.B., Batch process management. Chem. Eng. Prog., 1990, 86(6), 66-77.

[54] Dach R., Song J.J., Roschangar F., Samstag W., Senanayake C.H., The Eight Criteria Defining a Good Manufacturing Process. Org. Process Res. Dev., 2012, 16, 1697-1706.

[55] Toniolo S., Arico F., Tundo P., A comparative environmental assessment for the synthesis of 1,3-oxazin-2-one by metrics: greenness evaluation and blind spots. ACS Sustain. Chem. Eng., 2014, 2, 1056-1062. 